\title{
Compressive Cervical Myelopathy in X-Linked Hypophosphataemic Rickets
}

\author{
Conor Fearon, Adnan Abbas, Stephen A. Ryan, Sean J. Fitzgerald, Seamus Looby
}

Keywords: Rickets, Cervical Myelopathy, X-Linked Hypophosphataemic Rickets, Neuroimaging

doi:10.1017/cjn.2018.54

Can J Neurol Sci. 2018; 45: 568-570

A 45-year-old Caucasian man with X-linked hypophosphataemic rickets (XLHR), and no other risk factors for ossification of the posterior longitudinal ligament (OPLL), presented with a 3-week history of progressive right hand and leg weakness. This was noticed when weight-bearing, 5 weeks after a right total knee replacement. Examination revealed right upper limb and right lower limb weakness and bilateral lower limb spasticity with extensor plantar responses, compatible with a cervical mye- lopathy. CT cervical spine revealed coarse calcification of the posterior longitudinal ligaments (PLL), from C4 to C7, and of the ligamentum flavum (LF), from $\mathrm{T} 3$ to $\mathrm{T} 4$, causing severe central canal stenosis (Figure 1). MRI spine showed extensive abnormal ossification of the PLL at C2-C6, and confirmed compressive myelopathy of the cervical cord, with spinal cord oedema at this level (Figure 2). The patient declined surgery (Figure 2).

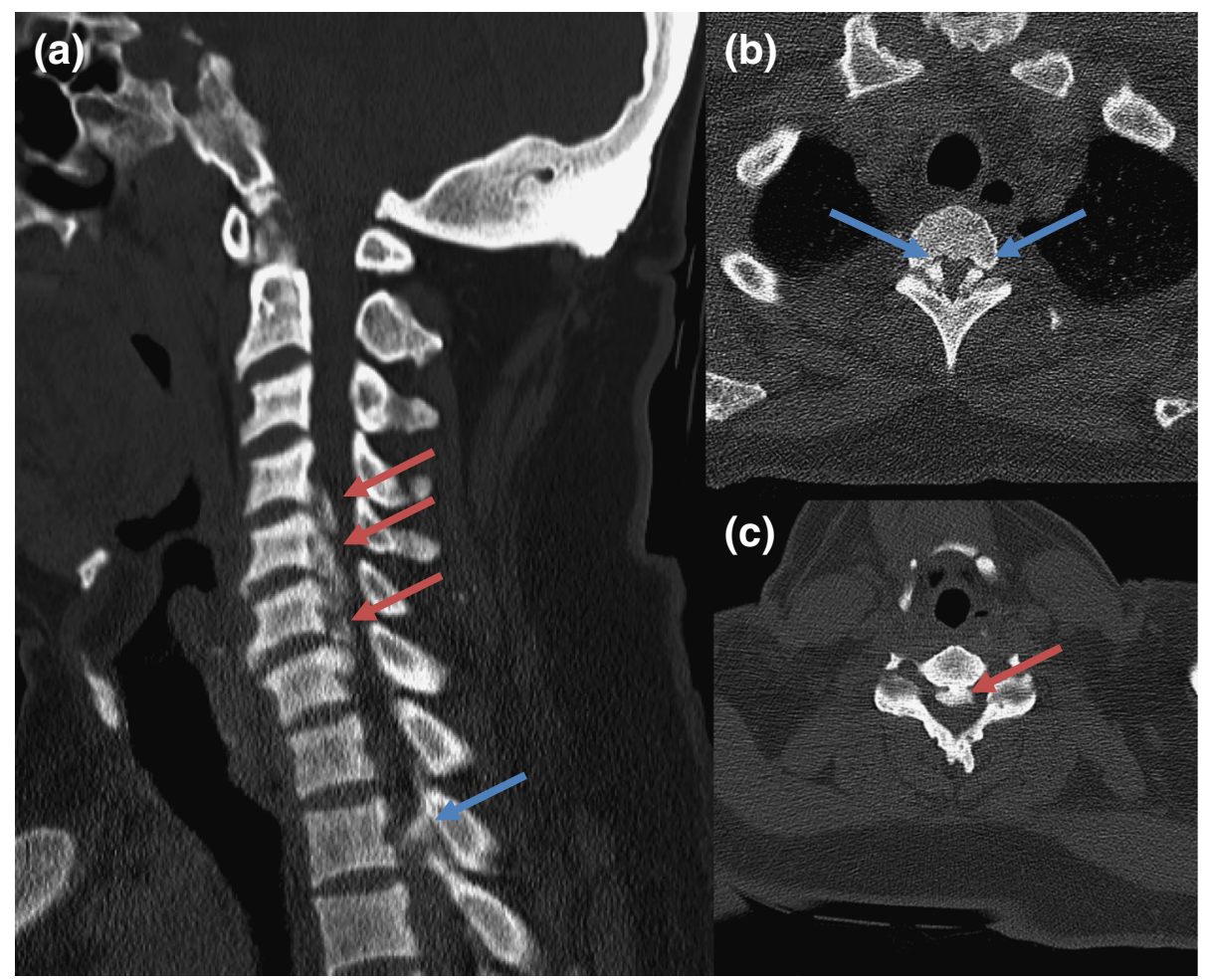

Figure 1: $C T$ cervical spine, sagittal (A) and axial $(B, C)$ : marked ossification of posterior longitudinal ligament (red arrows) and ligamentum flavum (blue arrows), causing severe central canal stenosis.

From the Department of Neurology, Beaumont Hospital, Dublin, Ireland (CF, AA, SAR, SJF); Department of Radiology, Beaumont Hospital, Dublin, Ireland (SL).
Received DeCEmber 18, 2016. Final Revisions Submitted March 14, 2018. DATE OF AcCEPTANCE ApRIL 12, 2018.

Correspondence to: Dr. C. Fearon, Department of Neurology, Beaumont Hospital, Dublin, Ireland. Email: cfearon@tcd.ie 


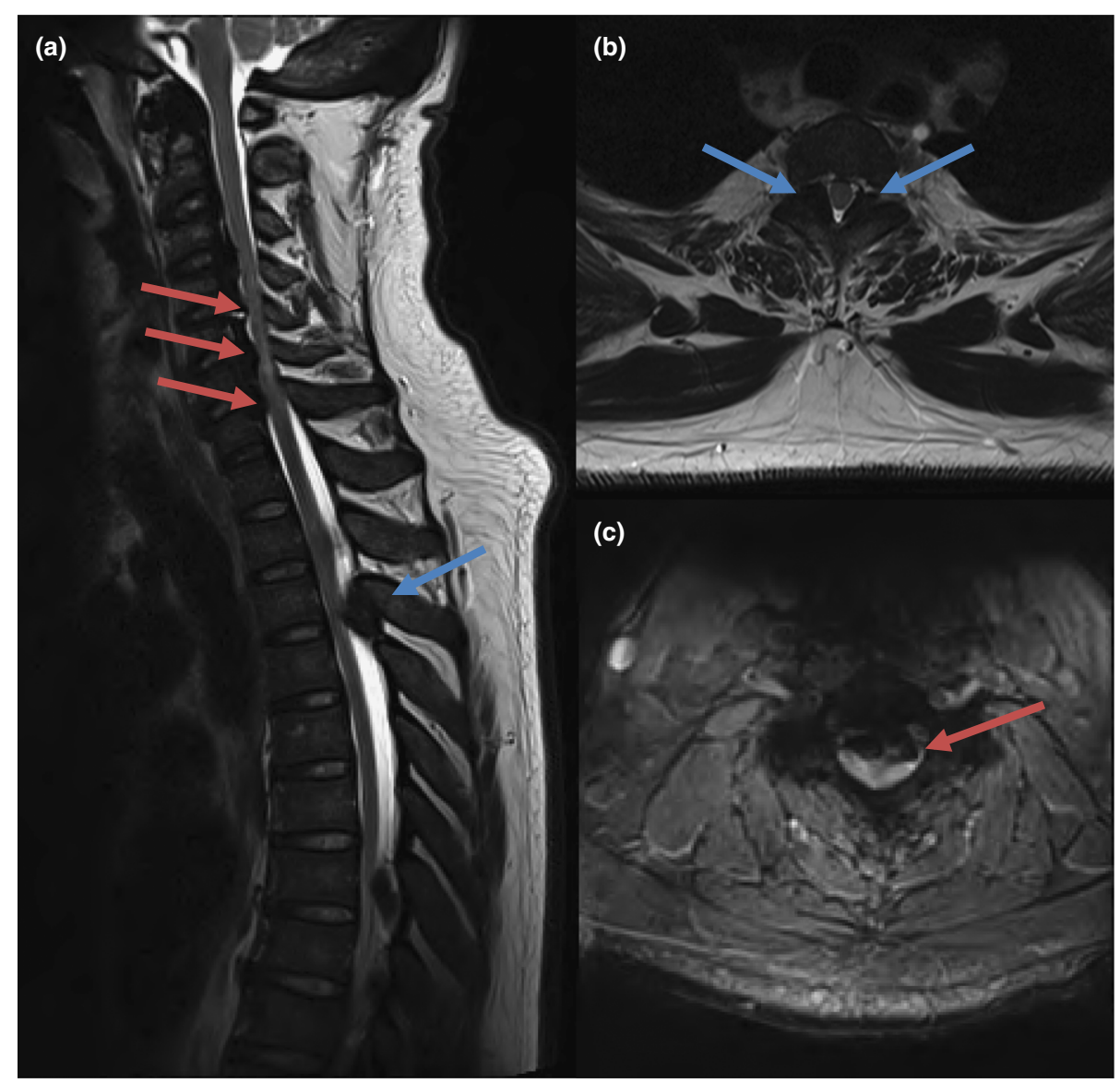

Figure 2: T2-weighted MRI spine, sagittal $(A)$ and axial $(B, C)$ : diffuse hypointense bone marrow signal abnormality (also shown on T1 sequence; likely a treatment effect), mass-like T2hypointensities within posterior longitudinal ligament at C4-C7 (red arrows), which causes cord oedema at these levels; focal and expanded T2-hypointensities in ligamentum flavum at T3-T4 and T7-T8 (blue arrows).

$\mathrm{X}$-linked hypophosphataemic rickets is a common genetic cause of hypophosphataemia due to mutations in the PHEX gene (Phosphate-regulating gene with Homologies to Endopeptidases on the X-chromosome). Affected individuals have a short stature that is usually limited to the lower extremities, with associated leg bowing. It is rarely a cause of spinal cord compression. Neuroradiological findings in XLHR reported in the literature include thickening of the vertebral laminae, facet joint hypertrophy, and ossification of the intervertebral discs, PLL, and/or LF. ${ }^{1}$

Ossification of the posterior longitudinal ligament is rare in Caucasians $(0.16 \%)$ compared with Asian populations $(2.4 \%){ }^{2}$ $\mathrm{CT}$ of the spine is the imaging modality of choice for detecting ossification of the PLL and LF. A recent case study showed evidence for the use of three-dimensional CT to be superior than lateral radiographic approaches in detecting small calcifications. ${ }^{3}$ Ossified lesions in MRI are hypointense on T1-weighted and T2-weighted images, which can be difficult to distinguish from the adjacent soft tissues and ligaments. MRI is generally superior to CT in detecting soft tissue abnormalities, such as concurrent disc protrusions and resultant spinal cord compression (as in this case). ${ }^{4}$ Radiographic predictors for the development of myelopathy in patients with OPLL include $>60 \%$ spinal canal stenosis; large range of motion of the cervical spine; and lateral deviatedtype OPLL. ${ }^{5}$ It is important to consider compressive myelopathy in patients with XLHR. We demonstrate the classical findings of ossification of the PLL and LF, leading to spinal cord compression.

\section{DISCLOSURES}

CF, AA, SAR, SJF, and SL do not have anything to disclose.

\section{Statement of AUthorship}

$\mathrm{CF}$ prepared the first draft of the manuscript and helped with the corrections. AA, SAR, and SJF reviewed the manuscript and helped with the corrections. SL reviewed the manuscript, and was involved in corrections, image selection, and description.

\section{REFERENCES}

1. Soehle M, Casey AT. Cervical spinal cord compression attributable to a calcified intervertebral disc in a patient with $\mathrm{X}$-linked hypophosphatemic rickets: case report and review of the literature. Neurosurgery. 2002;51(1):239-42; discussion 243-3. 
2. Choi B-W, Song K-J, Chang H. Ossification of the posterior longitudinal ligament: a review of literature. Asian Spine J. 2011; 5(4):267-76.

3. Izumi T, Hirano $\mathrm{T}$, Watanabe $\mathrm{K}$, et al. Three-dimensional evaluation of volume change in ossification of the posterior longitudinal ligament of the cervical spine using computed tomography. Eur Spine J. 2013;22(11):2569-74.
4. Tan WQ, Siew B, Wong S. Clinics in diagnostic imaging (161). Cervical OPLL with cord compression. Singapore Med J. 2015; 56(7):373-8.

5. Matsunaga S, Nakamura K, Seichi A, et al. Radiographic predictors for the development of myelopathy in patients with ossification of the posterior longitudinal ligament: a multicenter cohort study. Spine. 2008;33(24):2648-50. 